\title{
Burn injuries caused by e-cigarette explosions: A systematic review of published cases
}

\author{
Christopher M. Seitz' ${ }^{1}$ Zubair Kabir²
}

\begin{abstract}
INTRODUCTION E-cigarettes have the potential to cause burns from batteries that explode. Although e-cigarette explosion burns have been reported by the media (e.g. local online news, blogs), there is a need for a comprehensive review of published medical case reports regarding these injuries.

Methods CINAHL and PubMed were systematically searched using common terms regarding e-cigarettes (electronic cigarette, e-cigarette, vape, vaping, electronic nicotine delivery systems) in every combination with the term 'explosion'. Peerreviewed articles were included if they: were written in English, described case reports of burn injuries caused by e-cigarette explosions, and were published in any year. Cases were categorized by demographics, location of the e-cigarette explosion, burned body areas, types of burns, total body surface area of burns, the need for skin grafting, and the length of hospital stay.

RESULTS Thirty-one articles were included in the review and described 164 cases. Most patients (90\%) were male and between 20 to 29 years old. In the majority of cases $(65 \%)$, e-cigarettes exploded in pockets, compared to exploding in the face or hand. Common burned areas included the thigh, hand, genitals, and face. Burn severity was typically second-degree burns (35\%) or a combination of seconddegree and third-degree burns $(20 \%)$. In all, 48 patients required skin grafting, with 19 reporting a median hospital stay of 5 days.

CONCLUSIONS This review has several implications, including the need for regulation of batteries, education regarding battery safety, and leveraging images of the severity of e-cigarette explosion burns to discourage the use of e-cigarettes.
\end{abstract}

AFFILIATION

1 Department of Health \& Exercise Science, Appalachian State University, Boone, North Carolina, United States 2 School of Public Health, University College Cork, Ireland

CORRESPONDENCE TO

Christopher M. Seitz. Department of Health \& Exercise Science, Appalachian State University, 111 Rivers Street, PO Box 32071, 28608 Boone, North Carolina, United States.Email: seitzcm@ appstate.edu

\section{KEYWORDS}

burn, injury, explosion, e-cigarettes

Received: 29 May 2018 Revised: 15 August 2018 Accepted: 28 August 2018

\section{INTRODUCTION}

Electronic cigarettes (e-cigarettes) are a recent innovation. In 2003, the e-cigarette was invented by a Chinese pharmacist, and by 2010 several brands were established in the United States market ${ }^{1}$. Although three generations of e-cigarette designs have emerged over time, the basic components of e-cigarettes include: a battery, a reservoir for holding an e-juice (a solution that usually contains nicotine, flavorants, and propylene glycol and/or glycerin as a solvent), a heating element, and a mouthpiece. Specifically, the battery powers the heating element to heat the e-juice into an aerosol, which is then inhaled through the mouthpiece ${ }^{1}$.

E-cigarettes are growing in popularity, especially among adolescents and young adults in the United States. In 2015 , a total of $24 \%$ of high school students had used e-cigarettes at least one time in the past 30 days $^{2}$, a $900 \%$ increase $^{1}$ since 2011 . Moreover, data from the 2013-2014 National Adult Tobacco Survey indicated that $13 \%$ of young adults, age 18 to 24 years, were current users. The prevalence of e-cigarette use among these age groups resulted in a major report and call for action by the United States 


\section{Surgeon General ${ }^{1}$.}

The public health concern regarding e-cigarettes has typically focused on the chemicals that are potentially harmful (e.g. aerosols, formaldehyde, acetaldehyde, carcinogens, nicotine $)^{1}$, however, another health issue is bodily harm caused by e-cigarettes that explode ${ }^{3}$. Although the cause of these explosions has not been fully determined ${ }^{3}$, previous incidents suggest that overheating of the battery (also known as 'thermal runaway') may lead to explosions ${ }^{4,5}$, which has also explained the explosions observed from other devices using lithium-ion batteries (e.g. smart phones, laptops $)^{6}$. From 2009 to 2016, there were 195 e-cigarette explosions in the United States reported by the media, resulting in 133 injuries, with burns the most common injury ${ }^{4}$. Moreover, in 2016 alone, an estimated 1007 e-cigarette burn injuries were treated in emergency departments across the United States ${ }^{7}$.

It is also worth noting that besides burns, other cases of e-cigarette explosions involved a variety of serious injuries. Such injuries were inflicted to the hands ${ }^{8,9}$, face ${ }^{10}$, eyes ${ }^{11-13}$, mouth (e.g. lacerations, broken/missing t eeth $)^{8,11,14-20}$, and also resulted in broken neck bones ${ }^{21}$. In addition, the first-ever death from an e-cigarette explosion was reported in 2018 , which was due to e-cigarette shrapnel entering the person's brain ${ }^{22}$.

Given the prevalence of e-cigarette explosions, there is a need for a comprehensive review of published case reports in peer-reviewed journals regarding injuries, especially burns. Although previous studies have reviewed the literature regarding burns caused by e-cigarette explosions, these reviews were not exhaustive of the literature ${ }^{23-26}$, or focused only on explosion injuries reported by the media (e.g. online news, television, blogs $)^{4,27}$ instead of on the scientific literature. In addition, these reviews have been disseminated through journals that are meant for physicians specializing in burn injuries, as opposed to health professionals dealing with tobacco prevention and cessation. As such, the purpose of this study was to provide a systematic review of published medical case reports of burn injuries caused by e-cigarette explosions in peer-reviewed journals.

\section{METHODS}

Articles were identified by using a comprehensive list of search terms and relevant databases. During January 2018, the Cumulative Index to Nursing and Allied Health Literature (CINAHL) and PubMed were searched using common terms regarding e-cigarettes (electronic cigarette, e-cigarette, vape, vaping, electronic nicotine delivery systems) and in every combination with the term 'explosion'. To locate additional articles, 'berry picking' methods of 'backward chaining' ( searching through the reference pages of relevant articles) and also 'forward chaining' ( searching the studies that cited relevant articles $)^{28}$ were used in Web of Science and Google Scholar ${ }^{29,30}$.

The resulting articles were included in the literature review based on the following eligibility criteria: written in English, described case reports of burn injuries caused by e-cigarette explosions, published in peer-reviewed journals, and published in any year. Articles were excluded if they described injuries other than burns or burns that were due to reasons other than an explosion (i.e. burns from a hot e-cigarette battery that did not explode). The inclusion and exclusion of articles were documented by the recommended flow diagram of the Preferred Reporting Items for Systematic Reviews and MetaAnalyses (PRISMA) ${ }^{31}$.

The articles included in the review were then analyzed. Cases were categorized by demographics, location of the e-cigarette explosion, burned body areas, types of burns, total body surface area of burns, the need for skin grafting, and the length of hospital stay.

\section{RESULTS}

A total of 31 articles ${ }^{8,9,11,13,16-18,20,23,25-27,32-50}$ met the review's inclusion criteria (Figure 1). These were published from 2015 to 2017 in a variety of medical journals. In all, the articles described 164 cases. The explosions took place in the United States, the United Kingdom, Canada, Germany, and France.

The cases varied in terms of demographics. The majority of patients $(90 \%)$ were male. Of the reports that specified the patient's age, there was a range in age from 16 to 63 years $(M=1.3, M d n=29$, $\mathrm{SD}=10.5$ ) and mostly were young (20 to 29 years) (Table 1). 
Figure 1. Recommended PRISMA flow diagram for article inclusion

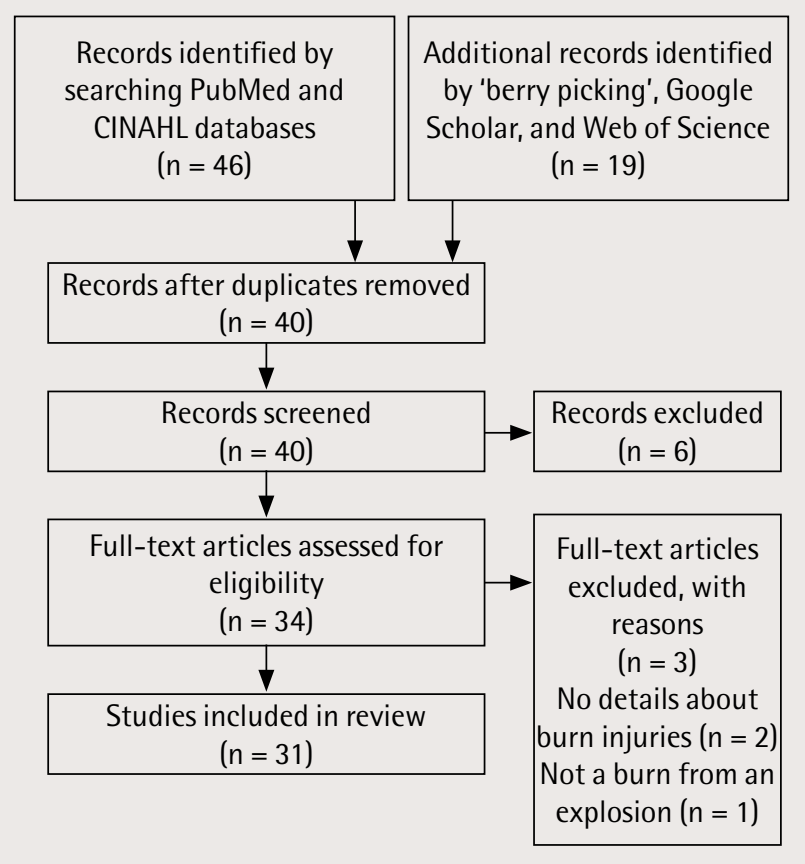

Table 1. Demographics of e-cigarette explosion cases

$\begin{array}{lc}\text { Demographics } & \text { Frequency }(\%) \\ \text { Sex } & \\ \text { Male } & 147(90) \\ \text { Female } & 12(7) \\ \text { NA } & 5(3) \\ \text { Age (years) } & \\ 16-19 & 9(5) \\ 20-29 & 38(23) \\ 30-39 & 22(13) \\ 40-49 & 14(9) \\ 50-59 & 3(2) \\ 60+ & 1(<1) \\ \text { NA } & 77(47)\end{array}$

The cases also varied in terms of the location of the e-cigarette explosions and in the location and types of burn injuries. In the majority of cases (65\%), e-cigarettes exploded in people's pockets (Table 2). The most common injured body areas included the thigh, hand, genitals, and face (Table $2)$. Burn severity was typically second-degree (35\%) and a combination of second-degree and thirddegree burns (20\%). Reports described 84 patients'
Table 2. Location of e-cigarette explosions and description of burn injuries

\begin{tabular}{lc} 
Burn characteristics & Frequency $(\%)$ \\
Location of explosion & $107(65)$ \\
Pocket & $20(12)$ \\
Face & $16(10)$ \\
Hand & $21(13)$ \\
NA & \\
Burned body areas & $125(76)$ \\
Thigh(s) & $65(40)$ \\
Hand(s) & $21(13)$ \\
Genitals & $21(13)$ \\
Face & $12(7)$ \\
Torso & $6(4)$ \\
Arm(s) & $3(2)$ \\
Buttocks & $3(2)$ \\
Feet & \\
Degree of burns & $1(<1)$ \\
1st degree & $50(49)$ \\
2nd degree & $33(20)$ \\
2nd \& 3rd degree & $13(8)$ \\
3rd degree & $59(36)$ \\
NA & \\
Total body surface area, $\%$ & $15(9)$ \\
$<1-5.6$ & \\
$6-10$ & \\
$11+$ & \\
NA & \\
\hline
\end{tabular}

total body surface area (TBSA) burns, which ranged from $0.5 \%$ to $27.25 \%(\mathrm{M}=4.9, \mathrm{Mdn}=4, \mathrm{SD}=4.3)$ (Table 2). In all, 48 patients required skin grafting. Of the articles that described 19 patients' length of stay in the hospital for treatment, the number of days ranged from 1 to 31 days $(\mathrm{M}=7.4, \mathrm{Mdn}=5$, $\mathrm{SD}=6.9$ ).

Several reports included information regarding the context of the explosion (Table 3 ). Several cases reported the e-cigarette touching metallic objects (e.g. coins, car keys) in the pocket during the time of explosion. Others stated issues related to charging their e-cigarette battery. Explosions took place while e-cigarettes were being used or while being stored in users' pockets. 


\section{Table 3. Precipitating events of e-cigarette explosion burn injuries}

\begin{tabular}{|c|c|c|c|}
\hline First author, year & Study location & Cases & Precipitating events \\
\hline Anderson, 2017 & USA (Kentucky) & 1 & The e-cigarette exploded during use \\
\hline Arnaout, 2017 & United Kingdom & 12 & Not specified \\
\hline Bauman, 2016 & USA (Arizona) & 3 & $\begin{array}{l}\text { One patient changed the e-cigarette's battery and then put the device in his pocket, } \\
\text { which contained car keys and coins. A different patient stated that his battery was } \\
\text { over 1-year old, but he claimed to charge it correctly }\end{array}$ \\
\hline Bohr, 2016 & Germany & 1 & $\begin{array}{l}\text { The patient stated that the e-cigarette (tank style) contained nicotine-free e-liquid } \\
\text { that was diluted with water }\end{array}$ \\
\hline Cason, 2016 & USA (Alabama) & 1 & Not specified \\
\hline Colaianni, 2016 & USA (Massachusetts) & 2 & The e-cigarettes exploded in both of the patients' pockets \\
\hline Gibson, 2018 & USA (Oregon) & 14 & $\begin{array}{l}\text { Of the patients, } 7 \text { reported that the e-cigarette device itself exploded, the other } 7 \\
\text { reported that a loose lithium ion battery exploded in their pockets }\end{array}$ \\
\hline Goverman, 2016 & USA (Massachusetts) & 5 & Not specified \\
\hline Harrison, 2016 & USA (South Carolina) & 1 & $\begin{array}{l}\text { The patient charged the e-cigarette's lithium-ion battery in a } 120 \text {-volt outlet using } \\
\text { an external charging device that was purchased separately from the e-cigarette }\end{array}$ \\
\hline Harshman, 2017 & Canada & 2 & The e-cigarette exploded in the patient's pocket, which also contained several coins \\
\hline Hassan, 2016 & United Kingdom & 6 & $\begin{array}{l}\text { All patients stored their batteries fully charged and close to other objects, including } \\
\text { cell phones and coins }\end{array}$ \\
\hline Herlin, 2016 & France & 2 & $\begin{array}{l}\text { One patient had a battery and device (separate from each other) in his pocket that } \\
\text { were touching metal keys }\end{array}$ \\
\hline Jablow, 2015 & USA (New Jersey) & 1 & $\begin{array}{l}\text { The patient denied any misuse of the e-cigarette and was not using it at the time of } \\
\text { the explosion }\end{array}$ \\
\hline Jiwani, 2017 & USA (Texas) & 10 & $\begin{array}{l}\text { Of the patients, } 7 \text { had their e-cigarettes explode in their pockets, } 1 \text { reported a } \\
\text { vaporizer explosion, } 1 \text { was using the e-cigarette when it exploded, and } 1 \text { reported } \\
\text { that the e-cigarette exploded during a motorcycle crash }\end{array}$ \\
\hline Kite, 2016 & USA (Virginia) & 1 & The e-cigarette (Lone Wulf Mechanical Mod cigarette) exploded in the patient's hand \\
\hline Kumetz, 2016 & USA (California) & 2 & $\begin{array}{l}\text { One patient was using the e-cigarette when it exploded. The other patient had the } \\
\text { e-cigarette explode in the pocket }\end{array}$ \\
\hline Maraqa, 2017 & USA (Michigan) & 8 & All of the patients' e-cigarettes exploded in their pockets \\
\hline Nicoll, 2016 & United Kingdom & 2 & $\begin{array}{l}\text { One of the patient's e-cigarette battery was charged overnight and was not damaged } \\
\text { or over-heated prior to the explosion }\end{array}$ \\
\hline Paley, 2016 & USA (Missouri) & 2 & $\begin{array}{l}\text { Both patients were using their e-cigarettes when they exploded. One of the } \\
\text { e-cigarettes was a vapor pen }\end{array}$ \\
\hline Patterson, 2017 & USA (Alabama) & 2 & $\begin{array}{l}\text { One patient's e-cigarette exploded in the pockets and the other patient's e-cigarette } \\
\text { exploded while being used }\end{array}$ \\
\hline Ramirez, 2017 & USA (California) & 30 & Some of the patients stated that the battery came in contact with a metallic object \\
\hline Rogér, 2016 & USA (New York) & 1 & The patient was using his e-cigarette when it exploded \\
\hline Satteson, 2017 & USA (North Carolina) & 1 & $\begin{array}{l}\text { The patient changed the battery of the e-cigarette (Dark Horse atomizer with a SMPL } \\
\text { Mec Mod battery device), and then the device quickly heated and exploded }\end{array}$ \\
\hline Schroeder, 2016 & USA (Pennsylvania) & 1 & The patient's e-cigarette exploded in his pocket \\
\hline Serror, 2017 & France & 10 & $\begin{array}{l}\text { Of the patients, } 8 \text { had the e-cigarette exploded in their pockets, and } 2 \text { patients' } \\
\text { e-cigarettes exploded while being used }\end{array}$ \\
\hline Shastry, 2016 & USA (California) & 1 & $\begin{array}{l}\text { The patient denied misuse of the e-cigarette, and also stated he was paid to test } \\
\text { products for an e-cigarette company, and that the cigarette he used was an } \\
\text { experimental model that was customizable and large. The e-cigarette exploded while } \\
\text { the patient was using it }\end{array}$ \\
\hline Sheckter, 2016 & USA (California) & 3 & Each patient reported that their e-cigarette exploded in their pockets \\
\hline Smith, 2017 & USA (Florida) & 10 & $\begin{array}{l}\text { Of the patients, } 1 \text { reported pouring nicotine liquid into the e-cigarette when it } \\
\text { exploded, } 2 \text { reported the e-cigarette exploded while they were driving, } 6 \text { reported } \\
\text { the e-cigarette exploding in their pockets, and } 1 \text { reported the e-cigarette exploding } \\
\text { in the hand }\end{array}$ \\
\hline Toy, 2017 & USA (California) & 25 & Of the patients, 18 reported the e-cigarette exploding in their pockets \\
\hline Treitl, 2017 & USA (Florida) & 3 & Each patient reported that their e-cigarette exploded in their pockets \\
\hline Walsh, 2016 & United Kingdom & 1 & $\begin{array}{l}\text { The patient reported charging his e-cigarette overnight and the battery being in } \\
\text { contact with keys in his pocket when it exploded }\end{array}$ \\
\hline
\end{tabular}




\section{DISCUSSION}

The primary aim of this study was to systematically review and synthesize evidence on case reports of burn injuries caused by e-cigarette explosions. A total of 31 studies reporting 164 patients were included in this systematic review, finding that e-cigarette explosions happened most often in pants pockets and among young men. The burn injuries were usually on the thighs and hands, and ranged in severity. To the best of our knowledge, this is the most comprehensive review on this topic. There are implications both in terms of public health prevention and future scientific research on this issue.

Although the scientific community is divided regarding the benefits or harms of e-cigarettes ${ }^{51,52}$, the findings from this literature review side directly with the potential harm of e-cigarettes, due to explosion injuries. The findings have important implications regarding regulation, public health advocacy, and education, as other experts have also emphasized $^{53,54}$.

First and foremost, e-cigarette manufacturers should invest in research and development to improve the safety features of e-cigarette batteries. Brown and Cheng ${ }^{55}$ of the FDA's Center for Tobacco Products have called for e-cigarette manufactures to investigate the unknown failure mechanisms and create standardized protocols for testing e-cigarettes. In addition, Brown and Cheng recommend that manufacturers prevent thermal runaway by using circuits that protect against overcharging, integrating cut-offs for thermal power, and using internal overpressure relief mechanisms ${ }^{55}$. By ensuring proper design in regard to safety, manufacturers can help to prevent e-cigarette explosions and consequent injuries.

Second, health professionals should advocate the regulation of e-cigarette batteries and the prohibition of e-cigarettes in areas that could cause major damage by explosions. For example, given the United States Food and Drug Administration's (FDA) new authority over the packaging, labeling, and sale of e-cigarettes ${ }^{56}$, the FDA could mandate for e-cigarette retailers to provide information about potential explosions to customers. Voicing concern about e-cigarettes from a public health perspective is vital for the implementation of needed policy. In addition, it is important to advocate the prohibition of e-cigarettes in places where they are currently being used that could cause larger explosions and injuries, such as in airplanes ${ }^{57,58}$.

Third, the findings indicate that public health professionals and e-cigarette manufactures need to improve efforts to inform with regard to safe handling practices for e-cigarette batteries. Although the FDA has created a webpage dedicated to tips for avoiding explosions (e.g. use device specific batteries and chargers, replace batteries if damaged or wet, keep e-cigarettes away from metal objects, do not charge batteries overnight $)^{3}$, research suggests that the FDA's webpage is not among the most searched sites regarding e-cigarette batteries ${ }^{59}$. Manufacturer websites and information included with e-cigarette packaging are often lacking information about battery explosions or not providing credible material to users ${ }^{59}$. The results from our review suggest that this information should be targeted towards groups which experience e-cigarette injuries most often, such as young males.

Finally, information and images of the severity of e-cigarette explosion burns could be used as leverage for prevention efforts. Research indicates that fear appeals using graphic images can be effective in improving attitudes and changing health behaviors $^{60,61}$. For instance, interventions that show images of smoking's impact on premature facial wrinkles can instill motives for smoking cessation ${ }^{62-63}$. Research suggests that knowledge of e-cigarette explosions may deter young people from using e-cigarettes ${ }^{67,68}$. As such, public health professionals should consider using graphic images of e-cigarette explosion injuries in educational materials to prevent or reduce e-cigarette use.

\section{Limitations}

The limitations of this study should be considered when interpreting this review's findings. First, it is obvious that the specific search terms and databases used in this review cannot be expected to locate every relevant published article. As such, the conclusions from the articles may not mirror all published research on this topic. Second, several of the studies did not include detailed information about each patient; therefore, the extent of all the patients' burns may not exactly reflect that of the review's findings. Finally, by only researching medical case studies, 
our findings do not cover people that experienced an explosion but did not visit a hospital for treatment.

\section{CONCLUSIONS}

This study was the first to systematically and thoroughly review published medical case reports on e-cigarette burn injuries. The severity of burns and the commonly injured locations (e.g. thigh, hands, genitals, face) strongly indicate a need for improved e-cigarette construction and for health professionals to advocate regulation of e-cigarette batteries, as well as insist on improved dissemination of information on explosions, for safety and prevention purposes.

\section{REFERENCES}

1. U.S. Department of Health and Human Services. E-Cigarette use among youth and young adults. A report of the Surgeon General. Atlanta, GA: U.S. Department of Health and Human Services, Centers for Disease Control and Prevention, National Center for Chronic Disease Prevention and Health Promotion, Office on Smoking and Health, 2016.

2. Kann L, McManus T, Harris WA, et al. Youth Risk Behavior Surveillance-United States, 2015. MMWR. 2016;65:1-174. doi:10.15585/mmwr.ss6506a1.

3. U.S. Food and Drug Administration. Tips to help avoid "vape" battery explosions. https:// www.fda.gov/TobaccoProducts/Labeling/ ProductsIngredientsComponents/ucm539362.htm . Accessed February 2018.

4. U.S. Fire Administration. Electronic cigarette fires and explosions in the United States 2009-2016. https:// www.usfa.fema.gov/downloads/pdf/publications/ electronic_cigarettes.pdf. Published December 2017. Accessed February 14, 2018.

5. Wang Q, Ping P, Zhao X, Chu G, Sun J, Chen C. Thermal runaway caused fire and explosion of lithium ion battery. J Power Sources. 2012; 208: 210-224. doi:10.1016/j.jpowsour.2012.02.038

6. Mankowski PJ, Kanevsky J, Bakirtzian P, Cugno S. Cellular phone collateral damage: A review of burns associated with lithium battery powered mobile devices. Burns. 2016; 42: e61-364. doi:10.1016/j.burns.2015.10.01

7. Corey CG, Chang JT, Rostron BL. Electronic nicotine delivery system (ENDS) battery-related burns presenting to US emergency departments, 2016. Inj Epidemiol. 2018; 5: 4. doi:10.1186/s40621-018-0135-1.

8. Kite AC, Le BQ, Cumpston KL, et al. Blast injuries caused by vape devices: 2 case reports. Ann Plast Surg. 2016;77:620-2. doi:10.1097/SAP.0000000000000875.

9. Satteson ES, Walker NJ, Tuohy CJ, et al. Extensive hand thermal and blast injury from electronic cigarette explosion: A case report. Hand. 2017;13:NP1-NP5. doi: 10.1177/1558944717744333

10. Vaught B, Spellman J, Shah A, Stewart A, Mullin D. Facial trauma caused by electronic cigarette explosion. Ear Nose Throat J 2017; 96:139-142.

11. Cason DE, Morgan DE, Pietryga JA. Injuries from an exploding e-cigarette: A case report. Ann Intern Med. 2016;165:678-9. doi:10.7326/L16-0023

12. Khairudin MN, Mohd Zahidin AZ, Bastion ML. Front to back ocular injury from a vaping-related explosion. BMJ Case Rep. 2016; pii: bcr2016214964. doi:10.1136/bcr-2016-214964.

13. Paley GL, Echalier E, Eck TW, et al. Corneoscleral laceration and ocular burns caused by electronic cigarette explosions. Cornea 2016;35:1015-8. doi:10.1097/IC0.0000000000000881.

14. Archambeau BA, Young S, Lee C, Pennington T, Vanderbeek C, Miulli D, Gulhane J, Neeki M. E-cigarette blast injury: Complex facial fractures and pneumocephalus. West J Emerg Med. 2016; 17: 805807. doi:10.5811/westjem.2016.7.31354.

15. Brooks JK, Kleinman JW, Brooks JB, Reynolds MA. Electronic cigarette explosion associated with extensive intraoral injuries. Dent Traumatol. 2017; 33:149-152. doi: 10.1111/edt.12293.

16. Colaianni CA, Tapias LF, Cauley R, et al. Injuries caused by explosion of electronic cigarette devices. Eplasty. 2016;16:ic9.

17. Harrison R, Hicklin D. Electronic cigarette explosions involving the oral cavity. J Am Dent Assoc. 2016;147:891-6. doi:10.1016/j.adaj.2016.03.018.

18. Kumetz EA, Hurst ND, Cudnik RJ, et al. Electronic cigarette explosion injuries. Am J Emerg Med. 2016;34:2252.e12252.e3. doi:10.1016/j.ajem.2016.04.010

19. Moore J, Mihalache G, Messahel A. "Exploding" electronic cigarette: A case report. Br J Oral Maxillofac Surg 2016; 54:1056-1057. doi:10.1016/j.bjoms.2016.05.020.

20. Rogér JM, Abayon M, Elad S, et al. Oral trauma and tooth avulsion following explosion of e-cigarette. J Oral Maxillofac Surg. 2016;74:1181-5. doi:10.1016/j.joms.2015.12.017.

21. Norii T, Plate A. Electronic cigarette explosion resulting in a C1 and C2 fracture: A case report. J Emerg Med. 2017; 52:86-88. doi:10.1016/j.jemermed.2016.08.010.

22. Fortin J. This may be a first: Exploding vape pen kills a Florida man. The New York Times. May 16, 2018. https://www.nytimes.com/2018/05/16/us/man-killedvape-explosion.html. Accessed July 10, 2018.

23. Harshman J, Vojvodic M, Rogers AD. Burns associated with e-cigarette batteries: A case series and literature review. CJEM. 2017;1:1-9. doi:10.1017/cem.2017.32.

24. Hua M, Talbot P. Potential health effects of electronic cigarettes: A systematic review of case reports. Prev Med Rep 2016;4:169-78. doi:10.1016/j.pmedr.2016.06.002.

25. Maraqa T, Mohamed MAT, Salib M, et al. Too hot for 
your pocket! Burns from e-cigarette lithium battery explosions: A case series. J Burn Care Res. 2017; doi:10.1093/jbcr/irx015.

26. Treitl D, Solomon R, Davare DL, et al. Full and partial thickness burns from spontaneous combustion of e-cigarette lithium-ion batteries with review of literature. J Emerg Med. 2017;53:121-5. doi:10.1016/j.jemermed.2017.03.031.

27. Patterson SB, Beckett AR, Lintner A, et al. A novel classification system for injuries after electronic cigarette explosions. J Burn Care Res. 2017;38:e95-e100. doi:10.1097/BCR.0000000000000471.

28. Booth A. Unpacking your literature search toolbox: On search styles and tactics. Health Info Lib J. 2008;25:3137. doi:10.1111/j.1471-1842.2008.00825.x.

29. Falagas ME, Pitsouni EI, Malietzis GA, et al. Comparison of PubMed, Scopus, Web of Science, and Google Scholar: Strengths and weaknesses. FASEB J. 2007;22:338-42. doi:10.1096/fj.07-9492LSF.

30. Levay P, Ainsworth N, Kettle R, et al. Identifying evidence for public health guidance: A comparison of citation searching with Web of Science and Google Scholar. Res Synth Methods. 2016;7:34-45. doi:10.1002/jrsm.1158

31. Moher D, Liberati A, Tetzlaff J, et al. The PRISMA group. Preferred Reporting Items for Systematic Reviews and Meta-Analyses: The PRISMA Statement. PloS Med. 2009;6:e1000097. doi:10.1371/journal.pmed.1000097

32. Anderson H, Richie C, Bernard AA. Surprisingly volatile smoking alternative: Explosion and burns as risks of e-cigarette use. J Burn Care Res. 2017;38:e884. doi:10.1097/BCR.0000000000000454

33. Arnaout A, Dewi F, Nguyen D. Re: Burn injuries from exploding electronic cigarette batteries: An emerging public health hazard. J Plast Reconstr Aesthet Surg. 2017;70:981-2. doi:10.1016/j.bjps.2017.02.021

34. Bauman ZM, Roman J, Singer M, et al. Canary in the coal mine-Initial reports of thermal injury secondary to electronic cigarettes. Burns. 2017;43:e38-42. doi:10.1016/j.burns.2016.09.024

35. Bohr S, Almarzouqi F, Pallua N. Extensive burn injury caused by fundamental electronic cigarette design flaw. Ann Burns Fire Disasters. 2016;29:231-3.

36. Gibson CJS, Eshraghi N, Kemalyan NA, et al. Electronic cigarette burns: A case series. Trauma. 2018; doi:10.1177/1460408617738812

37. Goverman J, Schulz JT. Thigh burns from exploding e-cigarette. Burns. 2016;42:1618. doi:10.1016/j.burns.2016.07.024

38. Hassan S, Anwar MU, Muthayya P, et al. Burn injuries from exploding electronic cigarette batteries: An emerging public health hazard. J Plast Reconstr Aesthet Surg. 2016;69:1716-8. doi:10.1016/j.bjps.2016.09.014

39. Herlin G, Bekara F, Bertheuil N, et al. Deep burns caused by electronic vaping devices explosion. Burns. 2016;42:1875-7. doi:10.1016/j.burns.2016.08.015
40. Jablow LM, Sexton RJ. Spontaneous electronic cigarette explosion: A case report. Am J Med Case Rep. 2015;3:934. doi:10.12691/ajmcr-3-4-1

41. Jiwani AZ, Williams JF, Rizzo JA, et al. Thermal injury patterns associated with electronic cigarettes. Int J Burns Trauma 2017;7:1-5.

42. Nicoll KJ, Rose AM, Khan MA, et al. Thigh burns from exploding e-cigarette lithium ion batteries: First case series. Burns 2016;42:e42-6. doi:10.1016/j.burns.2016.03.027

43. Ramirez JI, Ridgway CA, Lee JG, et al. The unrecognized epidemic of electronic cigarette burns. J Burn Care Res. 2017;38:220-4. doi:10.1097/BCR.0000000000000472.

44. Schroeder N, Beavers K, Yu A, et al. Electronic cigarettes and thermal injury: A case report on the dangers of vaping. Crit Care Med. 2016;44:579.

45. Serror K, Chaouat M, Legrand MM, et al. Burns caused by electronic vaping devices (e-cigarettes): A new classification proposal based on mechanisms. Burns 2017;44:544-8. doi: 10.1016/j.burns.2017.09.005.

46. Shastry S, Langdorf MI. Electronic vapor cigarette battery explosion causing shotgun-like superficial wounds and contusion. West J Emerg Med 2016;17:17780. doi:10.5811/westjem.2016.1.29410.

47. Sheckter C, Chattopadhyay A, Paro J, et al. Burns resulting from spontaneous combustion of electronic cigarettes: A case series. Burns Trauma 2016;4:35. doi:10.1186/s41038-016-0061-9.

48. Smith SL, Smith C, Cheatham M, et al. Electronic cigarettes: A burn case series. J Nurse Pract 2017; 13 : 693-9. doi:10.1016/j.nurpra.2017.09.003.

49. Toy J, Dong F, Lee C, et al. Alarming increase in electronic nicotine delivery systems-related burn injuries: A serious unregulated public health issue. Am J Emerg Med. 2017;35:1781-2. doi:10.1016/j.ajem.2017.05.029.

50. Walsh K, Sheikh Z, Johal K, et al. Rare case of accidental fire and burns caused by e-cigarette batteries. BMJ Case Rep. 2016; doi:10.1136/bcr-2015-212868.

51. Green LW, Fielding JE, Brownson RC. The debate about electronic cigarettes: Harm minimization or the precautionary principle. Annu Rev Public Health. 2018; 39: 189-191. doi: 10.1146/annurev-publhealth-102417-124810.

52. Warner KE. How to think - not feel - about tobacco harm reduction. Nicotine Tob Res 2018; doi:10.1093/ntr/nty084.

53. Meernik, C, Williams FN, Cairns BA, Grant EJ, Goldstein AO. Burns from e-cigarettes and other electronic nicotine delivery systems. BMJ 2016; 354:i5024. doi:10.1136/bmj.i5024.

54. Rose AM, Nicoll KJ, Quaba O, Lowrie AG. E-cigarettesbeware of the rocket in your pocket. BMJ 2016; 353: i2712. doi:10.1136/bmj.i2712.

55. Brown CJ, Cheng JM. Electronic cigarettes: Product characterisation and design considerations. Tob Control. 2014; 23: ii4-ii10. 
doi:10.1136/tobaccocontrol-2013-051476.

56. US Food and Drug Administration. Deeming tobacco products to be subject to the Federal Food, Drug, and Cosmetic Act, as amended by The Family Smoking Prevention and Tobacco Control Act; Restrictions on the sale and distribution of tobacco products and required warning statements for tobacco products. Fed Regist. 2016;81:28973-29106.

57. Stillman FA, Soong A, Zheng LY, et al. E-cigarette use in air transit: Self-reported data from US flight attendants. Tob Control. 2015;24:417-8. doi:10.1136/tobaccocontrol-2013-051514

58. Transportation Security Administration. Electronic cigarettes and vaping devices. https://www.tsa.gov/ travel/security-screening/whatcanibring/items/ electronic-cigarettes-and-vaping-devices. Accessed May 29, 2018.

59. Pepper JK, Cress MJM, Gammon DG, et al. Battery safety information and warnings on e-cigarette packages and online. Tob Regul Sci. 2018;4:605-13. doi:10.18001/TRS.4.1.7

60. Witte K, Allen M. A meta-analysis of fear appeals: Implications for effective public health campaigns. Health Educ Behav. 2000;57,591-615. doi:10.1177/109019810002700506

61. World Health Organization. Tobacco Free Initiative (TFI): Warn about the dangers of tobacco. http://www. who.int/tobacco/mpower/warn/en/. Accessed February 2018.

62. Flett K, Grogan S, Clark-Carter D, et al. Male smokers' experiences of an appearance-focused facial-ageing intervention. J Health Psychol. 2017;22:422-33. doi:10.1177/1359105315603477.

63. Grogan S, Flett K, Clark-Carter D. Women smokers' experiences of an age-appearance anti-smoking intervention: A qualitative study. Br J Health Psychol. 2010;14:175-86. doi:10.1348/2044-8287.002006.

64. Grogan S, Flett K, Clark-Carter D, et al. A randomized controlled trial of an appearance-related smoking intervention. Health Psychol. 2011;30:805-9. doi:10.1037/a0024745

65. Hysert PE Mirand AL Giovino GA. "At Face Value": Age progression software provides personalised demonstration of the effects of smoking on appearance. Tob Control. 2003;12:238. doi:10.1136/tc.12.2.238

66. Weiss C Hanebuth D Coda P. Aging images as a motivational trigger for smoking cessation in young women. Int J Environ Res Public Health. 2001;7:3499512. doi: 10.3390/ijerph7093499

67. Copeland Al, Peltier MR, Waldo K. Perceived risk and benefits of e-cigarette use among college students. Addict Behav. 2017;71:31-7. doi:10.1016/j.addbeh.2017.02.005

68. Taira DA, Seto JC, Masterson J, et al. Attitudes of collegeaged electronic cigarette users in Hawaii. Health Behav
Policy Rev. 2018;5:94-102. doi:10.14485/HBPR.5.1.10
ACKNOWLEDGEMENTS The authors would like to acknowledge and thank all the patients who agreed to take part in each article's case report or case series.

CONFLICTS OF INTEREST Authors have completed and submitted the ICMJE Form for Disclosure of Potential Conflicts of Interest and none was reported.

FUNDING

There was no source of funding for this research.

PROVENANCE AND PEER REVIEW

Not commissioned; externally peer reviewed. 